Chapter 7

\title{
Parameters Identification of Stochastic Nonstationary Process Used in Earthquake Modelling
}

\author{
Giuseppe Carlo Marano, Mariantonietta Morga and \\ Sara Sgobba
}

Additional information is available at the end of the chapter

http://dx.doi.org/10.5772/54891

\section{Introduction}

The design of structures resistant to seismic events is an important field in the structural engineering, because it reduces both the loss of lives and the economic damages that earthquakes can produce. The accuracy and the robustness of the design of structures resistant to seismic events are still not completely guaranteed. In order to define rules in the design codes to design earthquake-resistant structures, several scholars have investigated the probability of a seismic event to occur in a specific location and its characteristics, like the intensity and the return time (e.g. frequency). Indeed, the return time and the characteristics of the earthquakes occurring in a given area determine the dynamic loads exciting a structure built in that area for its whole lifetime. The structural response to ground motion is function of the seismological parameters of the area where the earthquake occurs and the structure is built, in addition to the kind of structure. The earthquake characteristics related to the seismological parameters that strongly influence the structural response are the earthquake intensity, the rupture type and the epicentral distance. This leads to define the seismic dynamic loads exciting a structure as function of these seismological parameters. Unfortunately, the seismological parameters are not very useful in structural design. Instead, peak amplitude, frequency content, energy content and duration of the event are the characteristics of the earthquakes useful to structural design.

To design strategic or complex structures and infrastructures resistant to earthquakes, the analysis of the dynamic time-history response of the structure to earthquake records is preferred to the response spectrum analysis. Indeed, the dynamic time-history response provides temporal information of the structural response that is essential in non-linear analysis of some kind of structures to estimate their level of damage. Some design codes indicate the 
use of real records of earthquake ground motions as input of the dynamic time-history analysis of the structural response. Unfortunately, the selection of natural earthquake accelerograms that adhere to some criteria, such as the response spectrum for some design scenarios, is difficult. Indeed, the number of occurred earthquakes recorded in a specific area or with some characteristics is often not sufficient, because a wide set of accelograms is required for the design of earthquakes-resistant structures. To overcome this difficulty some design codes allow the use of modified natural records (with changes either in the time domain or in frequency domain) or synthetic accelerograms in the dynamic time history-analysis of the structural response. Unlikely, other design codes preclude the use of artificial accelerograms for the dynamic time-history analysis of the structural response because of the difficulty to generate accelograms that adhere to criteria for some design scenarios [1]. The approach based on the natural accelerograms is prevailing, since a real recorded accelerogram properly processed is undeniably a realistic representation of the ground shaking that is occurred in a particular seismological scenario. On the other hand, the recorded accelerogram represents a past seismic event occurred in a specific area and not a future event that will occur in that area and cannot be predicted because of stochastic nature of the seismic ground motions. This is a further reason to generate artificial accelerograms for the structural design on the basis of a stochastic model.

Several scholars have proposed different methods to generate the synthetic accelograms, but nowadays no model is indicated in the design codes to generate the artificial records of seismic ground motions. Moreover, the design codes that allow the use of artificial accelograms prescribe that the mean response spectrum of the synthetic earthquake records has to match a given response spectrum within a given tolerance.

The complex nature of the release of seismic wave, their propagation in soil and the unpredictability of the earthquake occurrence make the stochastic-based approach the most suitable to model the earthquake ground motion. In that sense the earthquake occurring in a specific area is modelled as a stochastic process, so each recorded seismic ground motion is defined as a sample function of that stochastic process. The artificial accelerograms are also sample functions of the stochastic process modelling the earthquake occurring in that area: they represent the possible future seismic events. For this reason in the design phase of a complex structure the structural response to these artificial accelerograms is calculated.

The stochastic process modelling the seismic events occurring in an area is defined through the characteristics of the strong ground motions recorded in that area. Several scholars have presented methods to define the stochastic model to describe the seismic ground motion and simulate artificial earthquake records. Firstly, stationary filtered white noise model have been proposed to describe and simulate earthquakes [2,3]. The most known of these models is the Kanai-Tajimi model $[4,5]$. Some scholars have modified this model [6] or have proposed stationary multi-filtered white noise models, as the Clough-Penzien model [7]. The stationary filtered white noise models catch only the main frequency of the seismic waves that excite the structure and the bandwidth of the stochastic process. The stationary stochastic models generate artificial accelograms with constant amplitude, while the amplitude of the real accelograms is time-varying. To overcome this limit, several schol- 
ars have proposed non-stationary filtered white noise models to simulate the seismic ground motions. This kind of earthquake models is obtained from the product of a filtered stationary White Noise process and an envelope function dependent on the time (figure 1). In literature there are several different envelope functions: the research of a reliable envelope function to model the ground motion intensity has been the goal of many studies. Some of these functions are simple and deterministic, like the one proposed by Bolotin [1], others are complex. Jangid [8] has given an overview of different envelope functions. The main feature that distinguishes the envelope functions proposed in literature is its shape: it describes the temporal evolution of the amplitude of the ground shake (trapezoidal, double exponential, log-normal, etc.). The envelope functions have simple parametric forms and the values of the parameters are estimated from some characteristics of the earthquake records available for a specific area, like the duration of the strong ground motion, the energy of the seismic event and the kind of soil. Some studies have proposed envelope functions correlated with seismological parameters [9, 10, 11]. Unfortunately, these parameters are not significant and useful for the structural design. Previously Baker [12] has proposed a correlation of the ground motion intensity parameters used to predict the structural and geotechnical response.

In order to reproduce the temporal variation of the frequencies of the seismic input shaking the structure, evolutionary non-stationary stochastic model have been proposed. In these complex models the parameters of the stationary filtered stochastic process have a temporal variation. The temporal evolution of the frequency content of the accelograms is due to the different velocity of the $\mathrm{P}$ waves, $\mathrm{S}$ waves and surface waves that are released in the epicentre of the earthquake [13].

This study presented in this chapter proposes a new simple and effective deterministic envelope function that correlates the temporal variation of the amplitude of the seismic records to the most significant seismological parameters of the ones used in structural design: the PGA and the kind of soil. The shape of the proposed envelope function is based on the Saragoni and Hart's (SH) exponential function [14] with three parameters determined through an energetic criterion. This shape of the envelope function gives a very good agreement with the selected time-histories, as a numerical analysis shows hereafter. The proposed envelope function is calculated through a new procedure composed by two stages. In the first stage a deterministic pre-envelope mean function that is the real envelope of a set of selected earthquake records is estimated. The values of the two parameters of the envelope function for each selected accelerograms are estimated through an identification procedure. In the second stage a regression law for each parameter is estimated to generalize the results and to obtain values of the parameters of the envelope function useful in the seismic engineering.

The identification procedure of the parameters of the Modified Saragoni and Hart's function proposed here is based on the continuous energy release of the earthquake measured through the Arias Intensity (AI). 


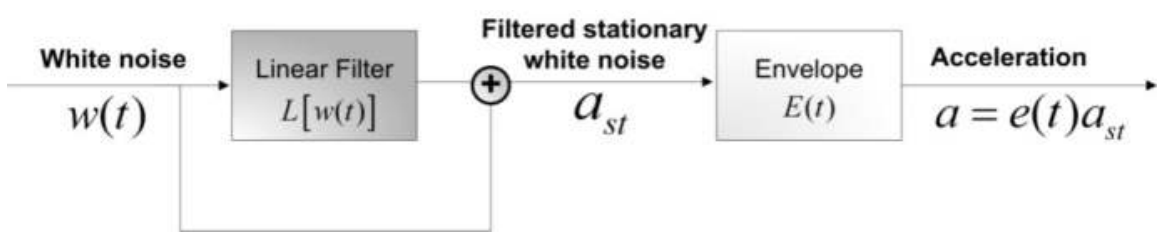

Figure 1. Scheme to simulate a stochastic ground motion

\section{Stationary filtered stochastic process modelling earthquakes}

As said in the introduction, a filtered stationary White Noise $(\mathrm{WN})$ process $w(t)$ is the simplest model of the ones proposed to represent the stochastic seismic acceleration process. In this kind of models the WN process models the acceleration at the bedrock. One or more filters model the action of the soil between the epicentre and the basement of the structure. Indeed the soil filters the seismic waves and the resulting filtered signal has the mean frequency content of the earthquake acceleration recorded on the ground surface. The most famous model of filtered WN process that defines the ground motion acceleration is the Kanai-Tajimi (K-T) model. In this model the filtering effect of the soil is defined by a SDoF system, characterized by two parameters: the damping ratio $\xi_{g}$ and the circular frequency $\omega_{g}$. The ground acceleration $a_{s t}$ exiting the structure is the absolute acceleration of the filter. Therefore, the differential equations of the K-T model are:

$$
\begin{gathered}
\ddot{x}+2 \xi_{g} \omega_{g} \dot{x}+\omega_{g}^{2} x=-w(t) \\
a_{s t}=\ddot{x}+w(t)=-\left(2 \xi_{g} \omega_{g} \dot{x}+\omega_{g}^{2} x\right) .
\end{gathered}
$$

This filter is a linear second order one, so the Power Spectral Density (PSD) function of the filtered $\mathrm{WN}$ is

$$
S(\omega)=S_{0} \frac{\left[1+\xi_{g}^{2}\left(\omega / \omega_{g}\right)^{2}\right]}{\left[1-\left(\omega / \omega_{g}\right)^{2}\right]^{2}+4 \xi_{g}^{2}\left(\omega / \omega_{g}\right)^{2}}
$$

where $S_{0}$ is the PSD of the WN process $[4,5,15]$. This model has some limits: the amplitude of the ground acceleration is constant and the frequency content is constant in time. The K-T model fails in simulating earthquake ground motion characterized by medium and long duration, because it is described by only two filter parameters, the damping ratio and the circular frequency. In order to overcome this limit, Clough and Penzien [7] have proposed a model characterized by a double filtered WN process. The two filters that define the effect of the soil between the bedrock and the surface are linear. The differential equations of the C-P model are: 


$$
\left\{\begin{array}{l}
a_{s t}(t)=\ddot{x}_{p}(t)=-\omega_{p}^{2} x_{p}(t)-2 \xi_{p} \omega_{p} \dot{x}_{p}(t)+\omega_{f}^{2} x_{f}+2 \xi_{f} \omega_{f} \dot{x}_{f}(t) \\
\ddot{x}_{p}(t)+\omega_{p}^{2} x_{p}(t)+2 \xi_{p} \omega_{p} \dot{x}_{p}(t)=\omega_{f}^{2} x_{f}+2 \xi_{f} \omega_{f} \dot{x}_{f}(t) \\
\ddot{x}_{f}(t)+2 \xi_{f} \omega_{f} \dot{x}_{f}(t)+\omega_{f}^{2} x_{f}=w(t)
\end{array}\right.
$$

where $x_{f}(t)$ is the response of the first filter characterized by the circular frequency $\omega_{f}$ and the damping ratio $\xi_{f}, x_{p}(t)$ is the response of the second filter characterized by the circular frequency $\omega_{p}$ and the damping ratio $\xi_{f}$ and $w(t)$ is the exciting $\mathrm{WN}$ process. In this model the stationary ground acceleration coincides with the acceleration of the second filter output: $a_{s t}(t)=\ddot{x}_{p}(t)$. In literature more complex models characterized by Multi-Degree of Freedom (MDoF) filters have be proposed to model seismic ground acceleration in a specific area. These models are defined by a larger number of parameters than the K-T and C-P models, so they model the soil filtering effect of the seismic waves better than the K-T and C-P models. On the other hand, these models with MDoF filters have a higher computational effort than the one of the K-T and C-P models. Some scholars $[13,16]$ have proposed non-stationary filtered WN models to take into account the temporal variation of the intensity of the acceleration records of the real earthquakes. The envelope function proposed in this study can be applied to modulate the amplitude in time of the stationary filtered White Noise obtained from a C-P model. Indeed, that model has four parameters correlated with the soil diffusion effect and reaches a good compromise between accuracy and computational effort to define seismic ground motion.

\section{Envelope function definition}

A Stationary stochastic process $\ddot{x}_{p}$ estimated through the C-P model is multiplied by an envelope function $E(t)$ to obtain a non-stationary filtered $\mathrm{WN}$ process with modulated intensity:

$$
a(t)=\ddot{x}_{p}(t) E(t)
$$

Each sample function of this non-stationary stochastic process is a synthetic accelerograms. The envelope function $E(t)$ proposed here is achieved from a complex procedure in two stages. A pre-envelope function defined from a set of selected recorded real accelerograms is estimated in the first step of the procedure. This pre-envelope function has the shape similar to the one developed by Saragoni and Hart [14] that is the most suitable for the set of the real records used. Indeed, this exponential deterministic envelope function has been chosen because it simulates better the strong ground motion than the other ones. It is continuous while other ones imply an arbitrary division into segments and the abrupt change in the frequency content of each segment. The Saragoni and Hart's (SH) function is

$$
\varphi(t)=\alpha t^{\eta} e^{-\beta t} \quad \alpha, \beta, \eta>0,
$$


where $\alpha, \beta$ and $\eta$ are three calibration parameters. In order to reduce the number of the parameters of the $\mathrm{SH}$ function from three to two, in this study the parameters $\alpha$ and $\beta$ are expressed as functions of the unknown time $t_{m}$ in which the $\mathrm{SH}$ function has its maximum value. The maximum value of the modulation function is estimated from the system of equations:

$$
\left\{\begin{array}{l}
\varphi\left(t_{m}\right)=1 \\
\dot{\varphi}\left(t_{m}\right)=0
\end{array}\right.
$$

From the solution of the eq. (7) the parameters $\alpha$ and $\beta$ are valuated as function of the other two parameters $\eta$ and $t_{m}$ :

$$
\begin{gathered}
\beta=\frac{\eta}{t_{m}} \\
\alpha=\left(\frac{e}{t_{m}}\right)^{\eta}
\end{gathered}
$$

Replacing the parameters $\alpha$ and $\beta$ in the SH function (6) with the expressions (8) and (9), the $\mathrm{SH}$ function becomes

$$
\varphi(\tau, \eta)=\tau^{\eta} e^{\eta(1-\tau)}
$$

where $\eta=t / t_{m}$ and the independent variables are only $\eta$ and $t_{m}$. In the follow this expression will be called Modified Saragoni and Hart (MSH) function. The intensity modulation of the earthquake ground motion is described not only by their peak value (Peak Ground Acceleration PGA), but also by the energy content or other quantities related to the energy content, such as the Arias Intensity (AI). The AI is defined as:

$$
I_{a}=\frac{\pi}{2 g} \int_{0}^{t} f \ddot{x}_{g}^{2}(t) d t
$$

and its mean value in stochastic terms is

$$
\mu\left[I_{a}\right]=\frac{\pi}{2 g} \int_{0}^{t_{f}}\left\langle\ddot{x}_{g}^{2}(t)\right\rangle d t=\frac{\pi}{2 g} \sigma_{\ddot{x}_{g}}^{2} \psi_{a}\left(t_{f}\right)
$$

where $t_{f}$ is total duration of the earthquake, $\ddot{x}_{g}(t)$ is the ground acceleration at time $t$ in one of the two horizontal directions, $g$ is acceleration due to gravity and the term $\psi_{a}\left(t_{f}\right)$ is defined by the expression

$$
\psi(a)=\int_{0}^{t} \varphi^{2}(\rho) d \rho .
$$




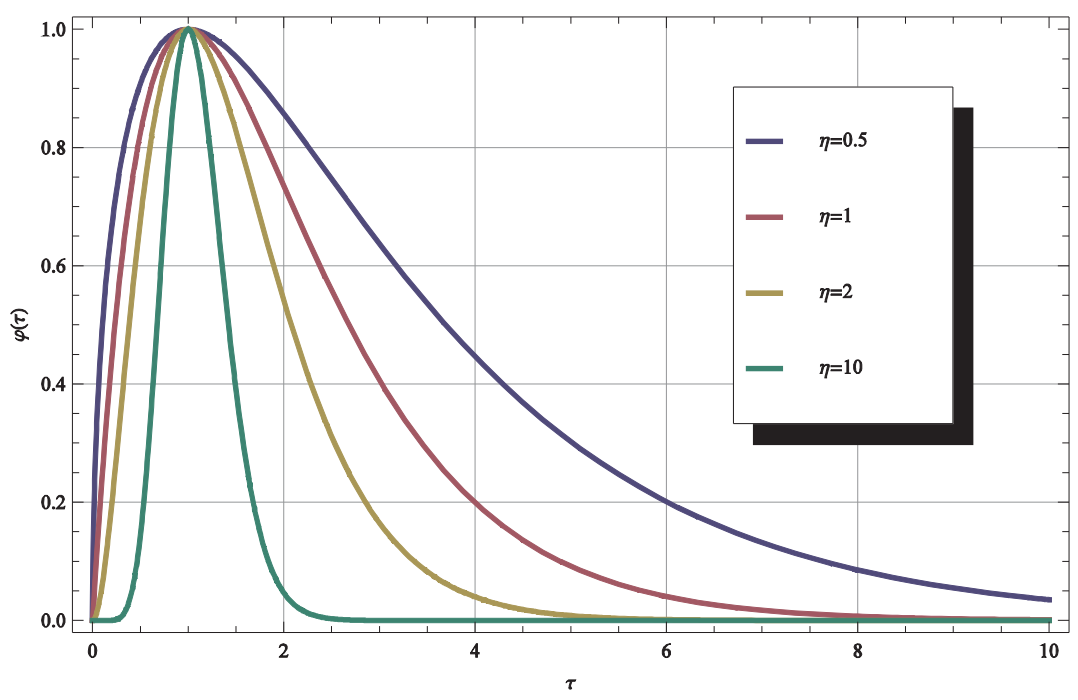

Figure 2. Sensitivity of the MSH function to the parameter $\eta$

Replacing the envelope function (10) in the expression (13), that expression becomes

$$
\int_{0}^{\tau}[\varphi(\rho, \eta)]^{2} d \rho=\tau^{2 \eta}\left(\left(\frac{e}{2}\right)^{2 \eta}(\eta \tau)^{-2 \eta} \Gamma(2 \eta)-\eta^{2 \eta} \tau E_{-2 \eta}(2 \eta \tau)\right)
$$

where $E_{n}(z)$ is the generalised exponential integral function

$$
E_{n}(z)=\int_{1}^{\infty} \frac{e^{-z}}{n} d=z^{n-1} \Gamma(1-n, z)
$$

while $\Gamma(z)$ is the Euler Gamma function

$$
\Gamma(z)=\int_{0}^{\infty} z-1 e^{-} d .
$$

The dimensionless ratio $\psi_{a}(\tau, \eta) / \psi_{a}\left(\tau_{f}, \eta\right)$ describes the energy release during earthquake event and it is plotted in the figure 3 for different value of the parameter $\eta$. This ratio is a function of the parameters $\tau$ and $\eta$. The parameter $\eta$ determines the velocity of the energy release during a seismic event, while the parameter $\tau$ describes the energy release during the duration of the event with respect to the time of maximum amplitude of the accelerograms. The values of the parameters $\tau$ and $\eta$ are estimated through an identification procedure. 


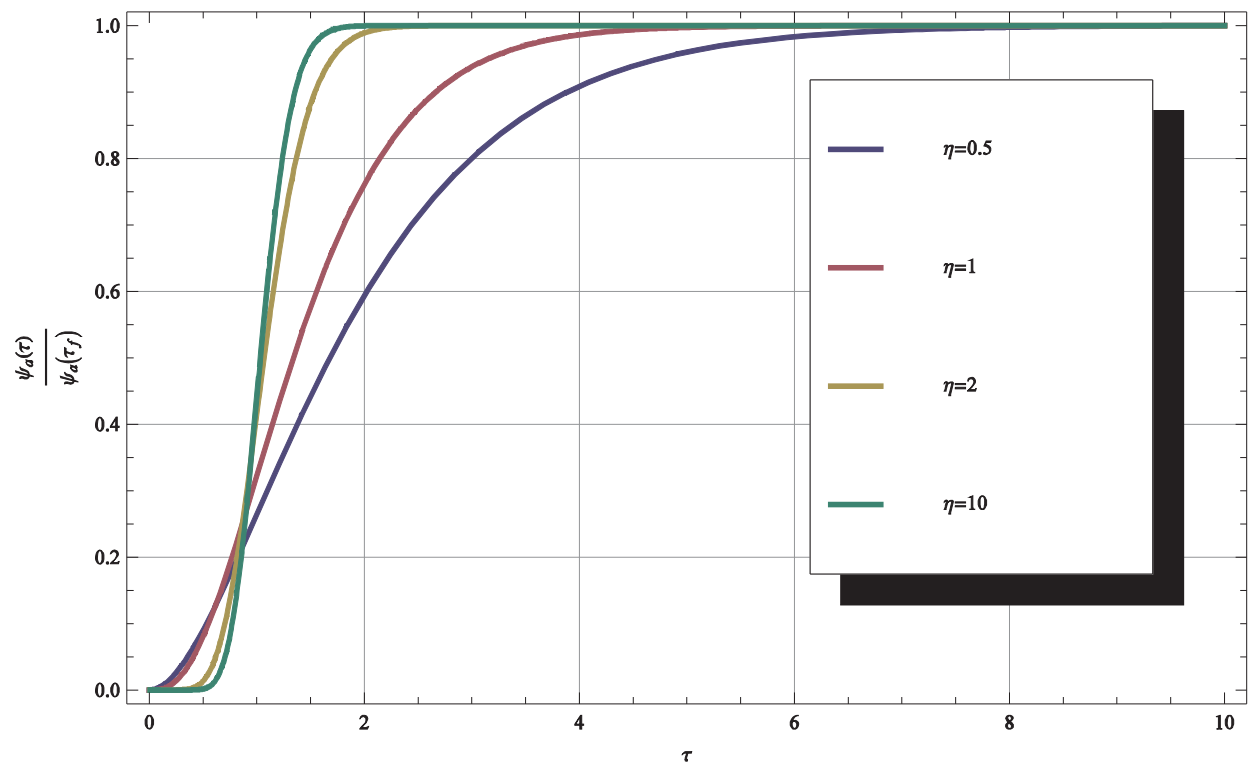

Figure 3. Sensitivity of the dimensionless ratio $\frac{\psi_{a}(\tau, \eta)}{\psi_{a}\left(\tau_{f}, \eta\right)}$ to the parameter $\eta$

\section{Numerical procedure to evaluate the parameters of the pre-envelope functions}

This section presents the identification procedure used to estimate the values of the parameters $\eta$ and $t_{m}$ that better characterize each of the selected real accelerograms of the PEER Next Generation Attenuation database.

The ground motion records of the PEER Next Generation Attenuation database that have been used in this study match the following criterion: the site where the seismic event is recorded has an average shear wave velocity in the top 30 meters comprised in four ranges according to the EC8 (B-C classes) and the NERPH classification (C-D classes) corresponding to stiff and soft soil respectively. The ground motion records of the PEER Next Generation Attenuation database are more than 7000 and half of them match this criterion. Further, the ground acceleration records of both the horizontal directions are used: for each selected accelogram of the database the weighted squared root of the sum of the squared east-west and northsouth components is calculated and after it is used to estimate the pre-envelope mean function and the PGA used in the procedure proposed here. 
The values of parameters $\eta$ and $t_{m}$ of the pre-envelope function are obtained by minimizing the difference of the ratios of the mean $\mathrm{AI}$ and the ratio of the term $\psi_{a}$ describing the energy release estimated for analytical expression and real earthquake record:

$$
\frac{\mu\left[I_{a}(t)\right]}{\mu\left[I_{a}\left(T_{f}\right)\right]}-\frac{\psi_{a}\left(t, t_{m}, \eta\right)}{\psi_{a}\left(T_{f}, t_{m}, \eta\right)}
$$
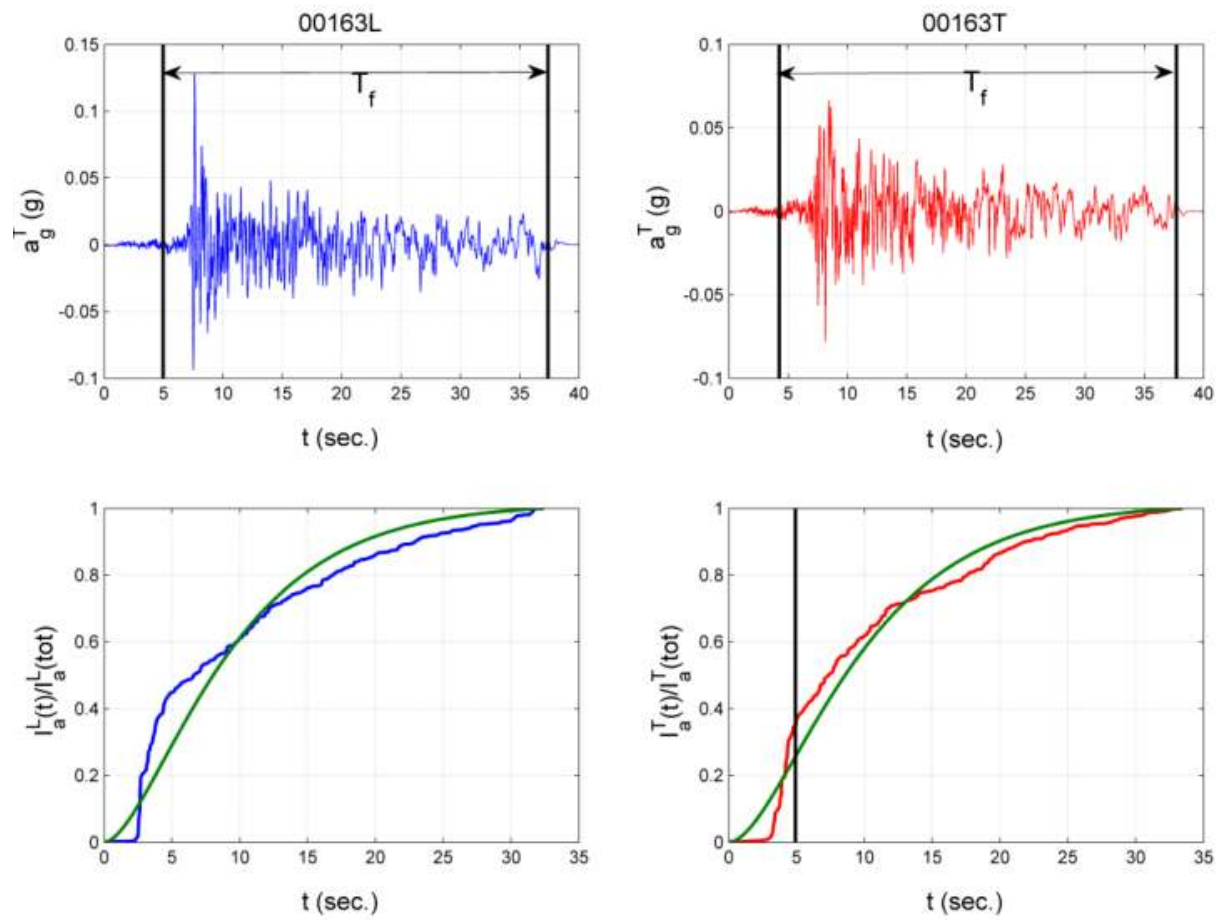

Figure 4. Record 00163 (kind of soil D) of the database and its Al. The blue lines indicates the L component and the red lines indicates the $T$ component of the real record of the seismic event and the green lines indicates the analytical Areas Intensity

The ratios of the equation to minimize are functions of the total duration time of the earthquake accelerogram $T_{f}$. The total duration time is defined as the time during which the $98 \%$ of the total energy of the seismic signal is released. It is evaluated as the time interval between the $1 \%$ and $99 \%$ of the AI of the seismic record. The total duration time is calculated for each selected earthquake record of the database. The values of the parameters $\eta$ and $t_{m}$ that describe the pre-envelope function for each real seismic event selected from the database seismic are achieved by means of an identification procedure formulated as an optimization problem. The Objective Function (OF) to minimize is 


$$
\text { OF }\left(\begin{array}{l}
- \\
b
\end{array}\right)=\frac{1}{T_{f}} \int_{0}^{T} T_{f}\left(\frac{I_{a}(\tau)}{I_{a}\left(T_{f}\right)}-\frac{\psi_{a}\left(\begin{array}{r}
- \\
\tau, b
\end{array}\right)}{\psi_{a}\left(\begin{array}{r}
T_{f}, b \\
)
\end{array}\right.}\right)^{2} d \tau,
$$

where $b=\left[t_{m}, \eta\right]$ is the vector of the variables. The optimization problem is solved by means of the Genetic Algorithm (GA) implemented in Matlab. To check the quality of this identification procedure of the parameters $\eta$ and $t_{m}$ for the pre-envelope function estimated for each earthquake record in the figures 4 and 5 the authors of this study have plotted a comparison between the AI of a real earthquake record and the analytical AI calculated using the parameters $\eta$ and $t_{m}$ estimated through identification procedure proposed. These two figures (4 and 5) show the comparison for two different earthquake records of the selected ones of the PEER Next Generation Attenuation database.
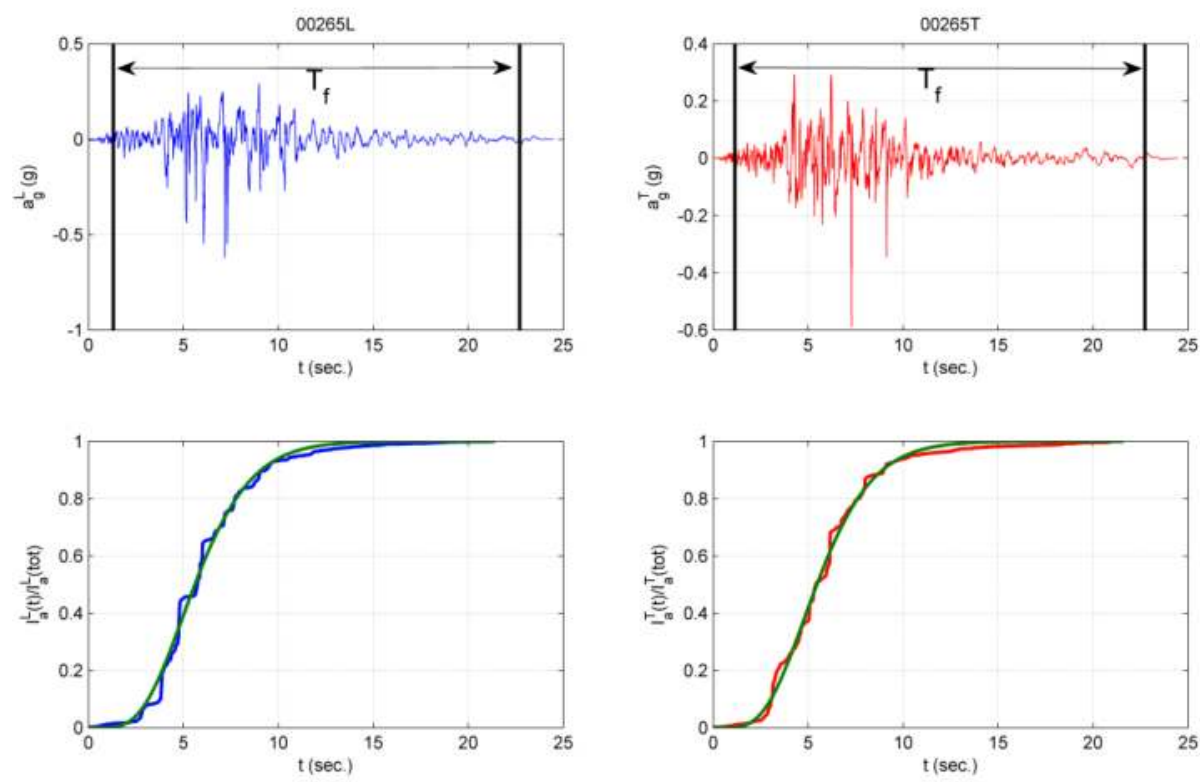

Figure 5. Record 00225 (kind of soil D) of the database and its Al. The blue lines indicates the L component and the red lines indicates the $T$ component of the real record of the seismic event and the green lines indicates the analytical Areas Intensity

The identification of the parameters of the pre-envelope function is applied to each selected earthquake record of the PEER Next Generation Attenuation database. The selected earthquakes records of the PEER Next Generation Attenuation database and their identified parameters $t_{m}$ and $\eta$ are grouped according to four types of soil, as afore said. After the identification of the parameters $\eta$ and $t_{m}$ the mean $\mathrm{AI}$ is calculated: 


$$
\mu\left[I_{a}(t)\right]=\frac{\pi}{2 g} \sigma_{\ddot{x}_{g}^{s s}}^{2} \psi_{a}\left(t, t_{m^{\prime}} \eta\right)
$$

A linear function is used to correlate the PGA with its stationary variance $\sigma_{\ddot{x}_{g}^{\text {st }}}$ at the time of maximum amplitude of the accelerogram $t_{m}$ :

$$
P G A=\kappa \sigma_{\ddot{x}}^{s t}
$$

where

$$
\kappa=P G A \sqrt{\frac{\pi}{2 g} \frac{\psi_{a}\left(t_{m}\right)}{\mu\left[I_{a}\left(t_{m}\right)\right]}} .
$$

The definition of the PGA is

$$
P G A=\max \left(\ddot{x}_{g}(\tau) \mid \tau\left[0, T_{f}\right]\right) \text {. }
$$

where $T_{f}$ is the total duration of the record. The normalized value of the PGA is

$$
\max \left(\ddot{x}_{g}^{N}\right)=1
$$

From the evaluation of (19), a new intensity measure $I_{e}$ called Envelope Intensity (EI) is introduced:

$$
I_{e}\left(T_{f}\right)=\frac{P G A}{g} \int_{0}^{T}{ }_{f} a_{g}^{N}(\tau) d \tau
$$

\section{Regression laws}

In the second stage of the procedure to evaluate the envelope function described by the PGA and the kind of soil the regression laws that relate the parameters of the proposed envelope function with the PGA are extracted. The parameters of the envelope function to be identified are the total duration time $T_{f}$, the AI $I_{a}$, the maximum envelope time $t_{m^{\prime}} \eta$ and $\kappa$. The regression laws of the envelope function obtained from the pre-envelope functions of the seismic event selected from the database are

$$
T_{f}(P G A)=T_{f}^{(0)}\left(\mathrm{e}^{\left(T_{f}^{(1)} P G A\right)}+\mathrm{e}^{\left(T_{f}^{(2)} P G A\right)}\right)
$$



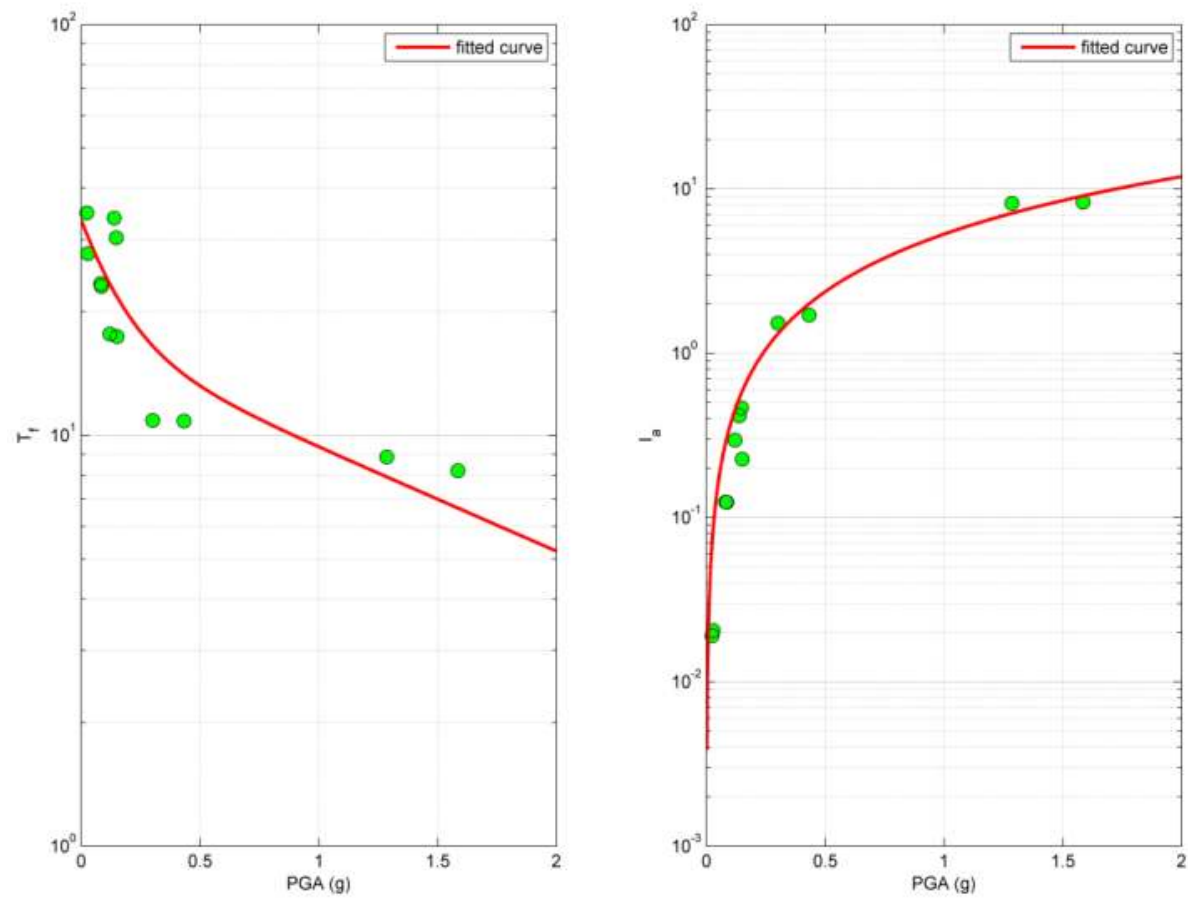

Figure 6. Values of $I_{a}$ and $T_{f}$ defined as function of the PGA. The value are related to the set of the seismic events selected from the database and occurred in sites characterized by kind of soil $A$.

$$
\begin{gathered}
I_{a}(P G A)=\exp \left(I_{a}^{0}+I_{a}^{1} \log (P G A)\right) \\
t_{m}(P G A)=t_{m}^{0} e^{\left(t_{m}^{1} P G A\right)} \\
\eta(P G A)=\eta^{0}+\alpha_{\eta} P G A \\
\kappa(P G A)=\kappa^{0}+\alpha_{\kappa} P G A .
\end{gathered}
$$

In these equations the PGA is express in $\mathrm{g}\left(9.81 \mathrm{~m} / \mathrm{sec}^{2}\right)$. In the figure $6,7,8$ and 9 it is fair that the curves of the regression law of the AI (eq. (26)) matches perfectly the trend of the AI 
valuated from the real data. The figures $6,7,8,9,10,11,12$ and 13 show that the curves of the regression laws for the other parameters do not fit perfectly the numerical values of these parameters estimated for the selected accelograms of the database. The regression laws achieve one purpose of this study: the definition of analytical relations to estimate the most important parameters for different kinds of soil that characterize the amplitude modulation of earthquake records and the energy release of seismic events. The numerical values of these parameters for the four kinds of soil are collected in the table 1. These results can be used to calculate the envelope function that modulates the amplitude intensity of stationary filtered $\mathrm{WN}$ process to generate artificial accelerograms typical of a certain kind of the soil.
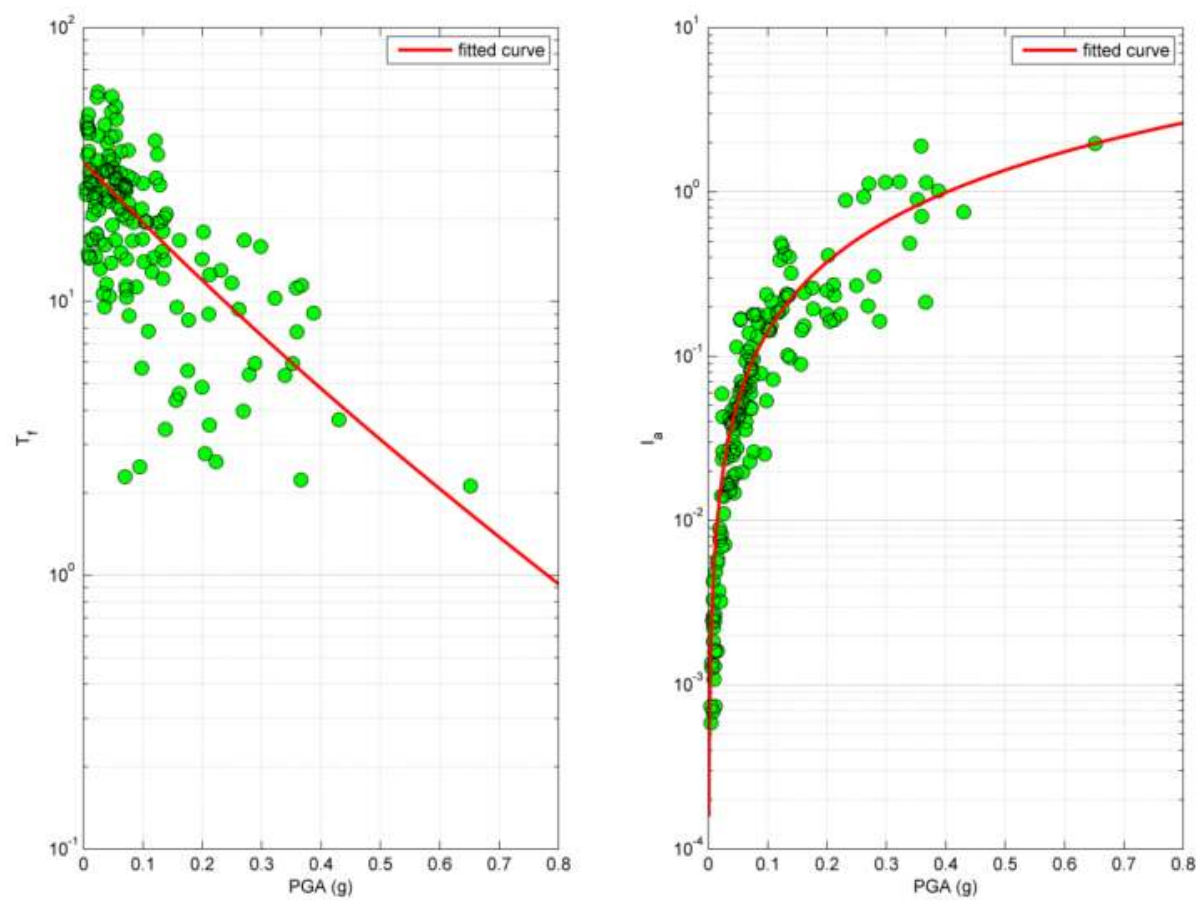

Figure 7. Values of $I_{a}$ and $T_{f}$ defined as function of the PGA. The value are related to the set of the seismic events selected from the database and occurred in sites characterized by kind of soil B. 


\begin{tabular}{|c|c|c|c|c|c|}
\hline Parameter & & Soil type A & Soil type B & Soil type C & Soil type D \\
\hline $\begin{array}{l}T_{f}^{0} \\
95 \% \text { confidence } \\
\text { bounds }\end{array}$ & {$[\mathrm{sec}]$} & $\begin{array}{l}16.73 \\
(9.371,24.09)\end{array}$ & $\begin{array}{l}16.12 \\
(14.03,18.2)\end{array}$ & $\begin{array}{l}9.7 \\
(8.189,11.21)\end{array}$ & $\begin{array}{l}14.76 \\
(12.13,17.4)\end{array}$ \\
\hline $\begin{array}{l}T_{f}^{1} \\
95 \% \text { confidence } \\
\text { bounds }\end{array}$ & {$[\mathrm{sec}]$} & $\begin{array}{l}-0.582 \\
(-1.496,0.332)\end{array}$ & $\begin{array}{l}-3.671 \\
(-10.21,-2.87)\end{array}$ & $\begin{array}{l}-10.32 \\
(-17.69,-2.957)\end{array}$ & $\begin{array}{l}-37.36 \\
(-66.02,-8.699)\end{array}$ \\
\hline $\begin{array}{l}T_{f}^{2} \\
95 \% \text { confidence } \\
\text { bounds }\end{array}$ & {$[\mathrm{sec}]$} & $\begin{array}{l}-6.367 \\
(-19.57,6.831)\end{array}$ & $\begin{array}{l}-6.724 \\
(-19.15,5.706)\end{array}$ & $\begin{array}{l}-0.7106 \\
(-1.316,-0.105)\end{array}$ & $\begin{array}{l}-1.057 \\
(-1.841,-0.2726)\end{array}$ \\
\hline $\begin{array}{l}I_{a}^{0} \\
95 \% \text { confidence } \\
\text { bounds }\end{array}$ & & $\begin{array}{l}1.668 \\
(1.556,1.78)\end{array}$ & $\begin{array}{l}1.28 \\
(1.092,1.469)\end{array}$ & $\begin{array}{l}1.543 \\
(1.467,1.618)\end{array}$ & $\begin{array}{l}1.463 \\
(1.351,1.575)\end{array}$ \\
\hline $\begin{array}{l}I_{a}^{1} \\
95 \% \text { confidence } \\
\text { bounds }\end{array}$ & & $\begin{array}{l}1.164 \\
(0.9277,1.4)\end{array}$ & $\begin{array}{l}1.407 \\
(1.247,1.567)\end{array}$ & $\begin{array}{l}1.568 \\
(1.418,1.717)\end{array}$ & $\begin{array}{l}1.424 \\
(1.312,1.537)\end{array}$ \\
\hline$t_{m}^{0}$ & [sec] & 11.37 & 15.56 & 5.379 & 6.412 \\
\hline$t_{m}^{1}$ & [sec] & -1.281 & -6.58 & -1.511 & -2.185 \\
\hline$\eta^{0}$ & & 1.798 & 3.4483 & 1.8058 & 1.8473 \\
\hline$a_{n}$ & & 0.874 & 3.4498 & 4.4542 & 1.9887 \\
\hline$\kappa^{0}$ & & 1.093 & 1.0446 & 1.6106 & 1.5914 \\
\hline$a_{\kappa}$ & & 0.652 & 2.0301 & 0.6132 & 0.5678 \\
\hline
\end{tabular}

Table 1. Parameters values of the regression laws estimated for all the four kinds of soil. 

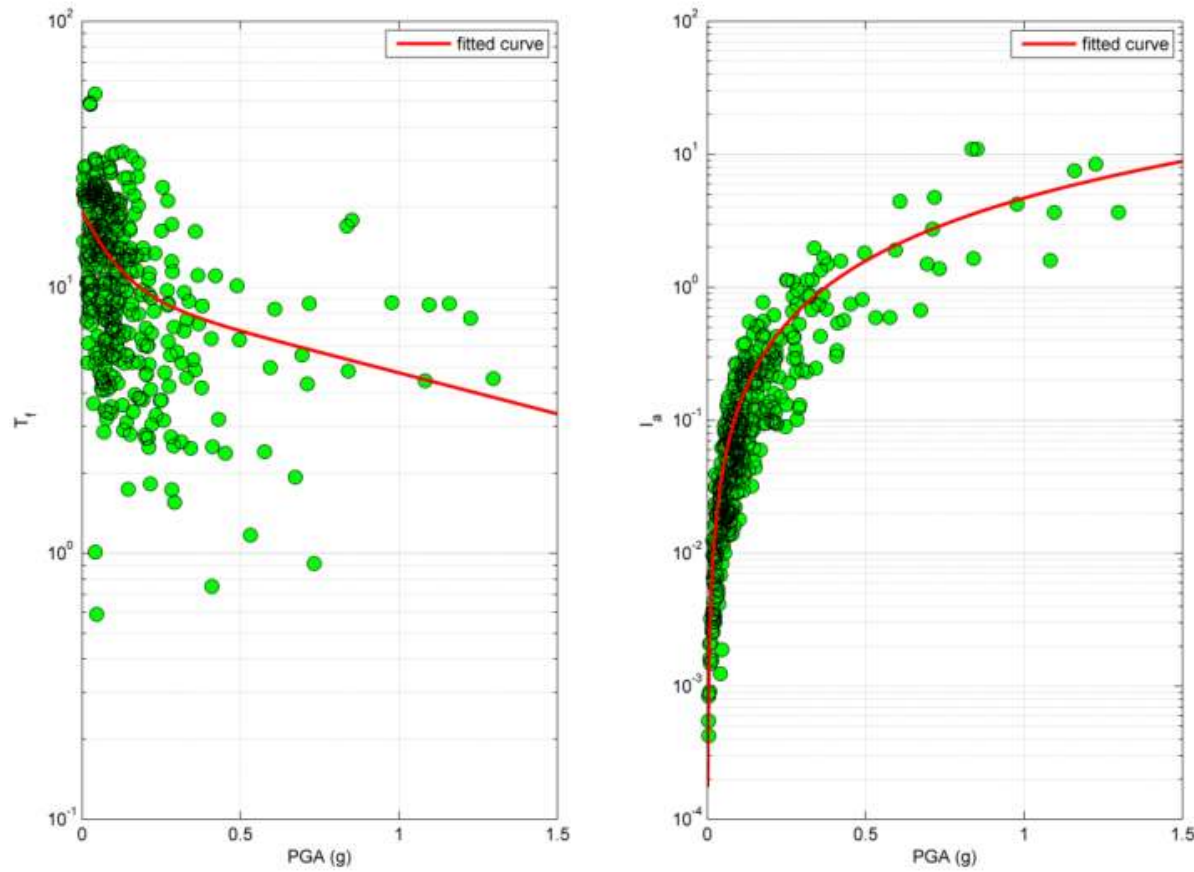

Figure 8. Values of $I_{a}$ and $T_{f}$ defined as function of the PGA. The value are related to the set of the seismic events selected from the database and occurred in sites characterized by kind of soil $C$ 

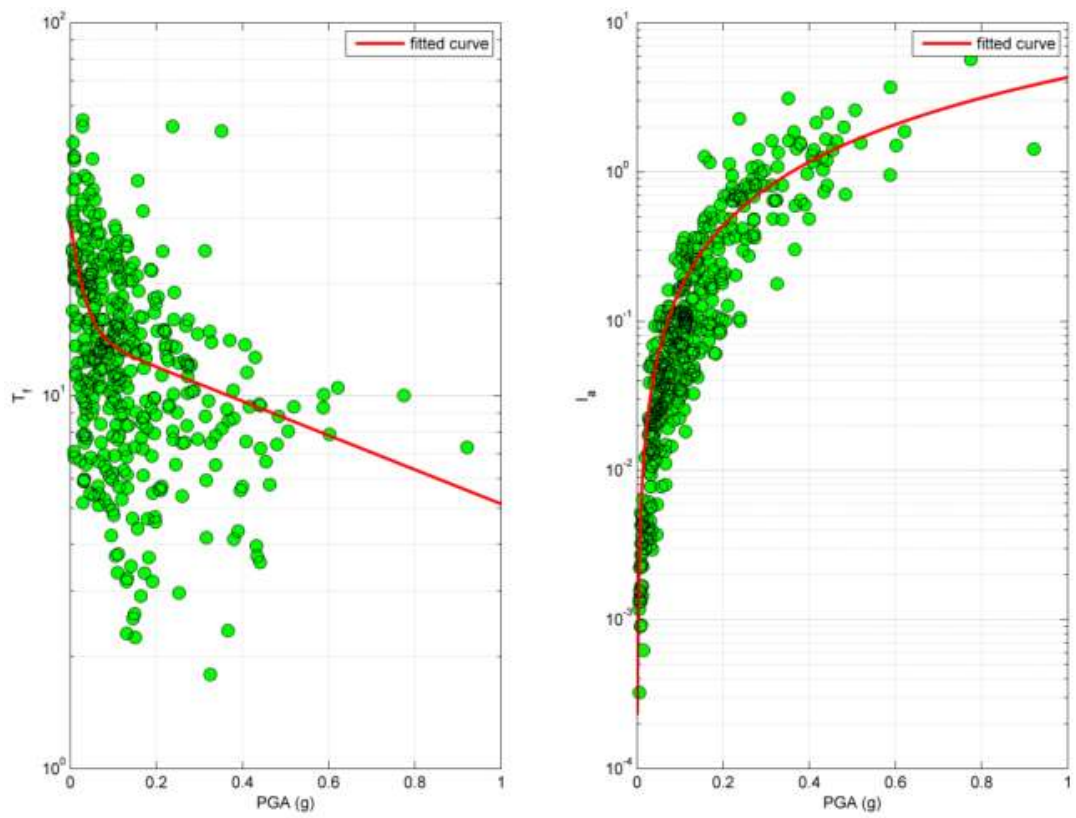

Figure 9. Values of $I_{a}$ and $T_{f}$ defined as function of the PGA. The value are related to the set of the seismic events selected from the database and occurred in sites characterized by kind of soil D.
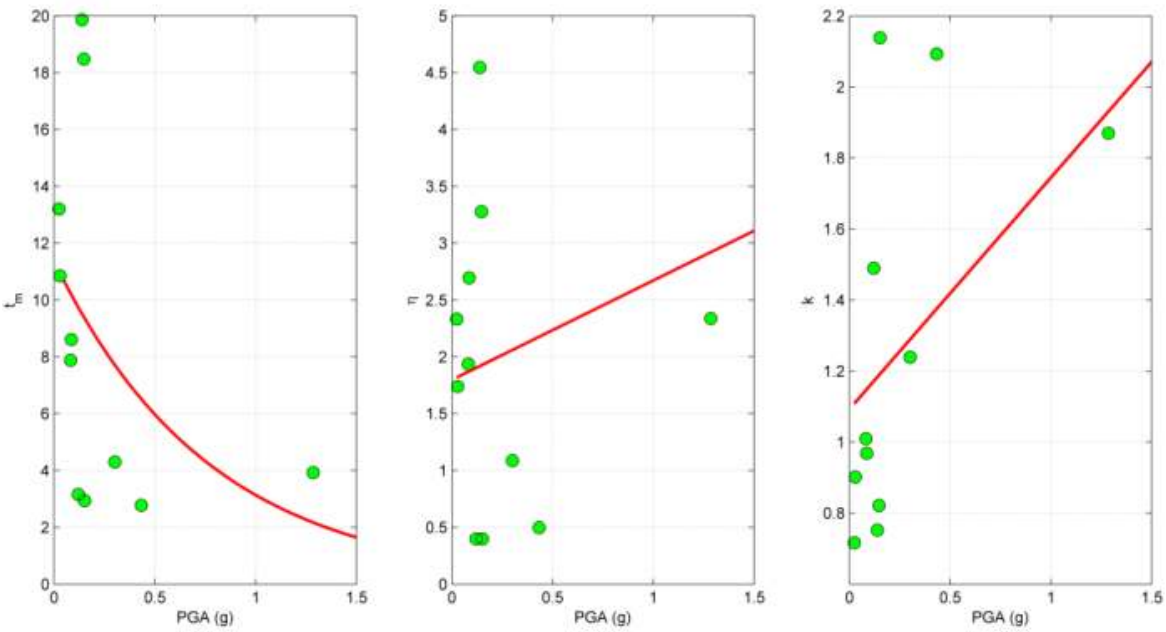

Figure 10. Values of $t_{m}, \eta$ and $k$ defined as function of the PGA. The value are related to the set of the seismic events selected from the database and occurred in sites characterized by kind of soil $A$. 

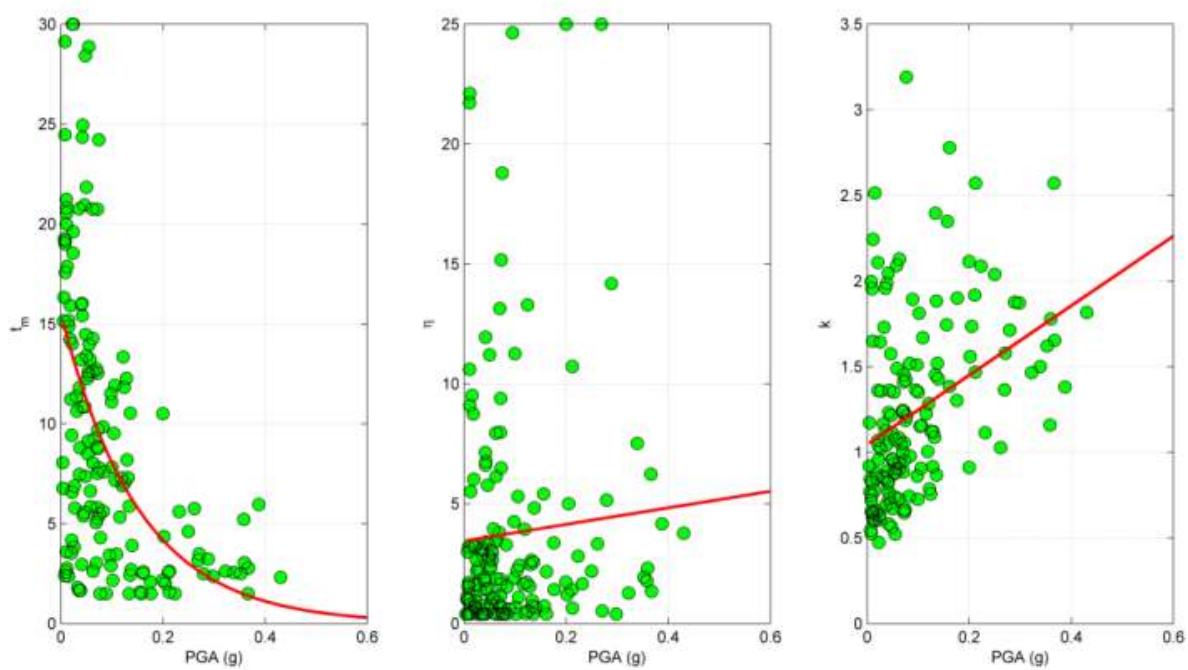

Figure 11. Values of $t_{m^{\prime}} \eta$ and $k$ defined as function of the PGA. The value are related to the set of the seismic events selected from the database and occurred in sites characterized by kind of soil B.
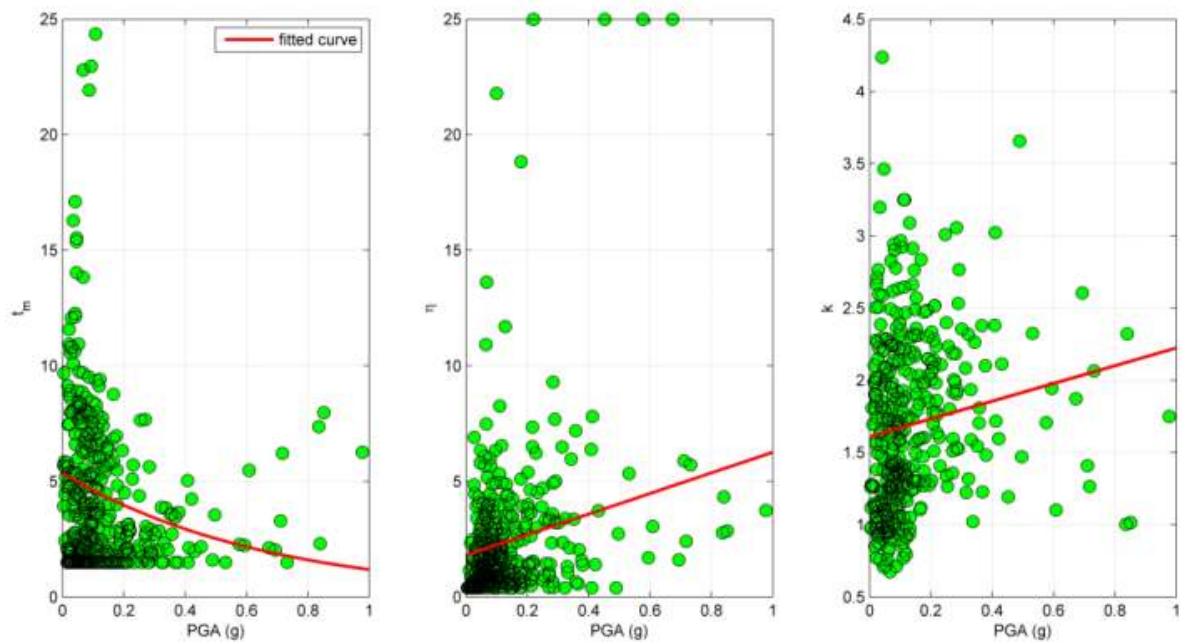

Figure 12. Values of $t_{m}, \eta$ and $k$ defined as function of the PGA. The value are related to the set of the seismic events selected from the database and occurred in sites characterized by kind of soil $C$. 

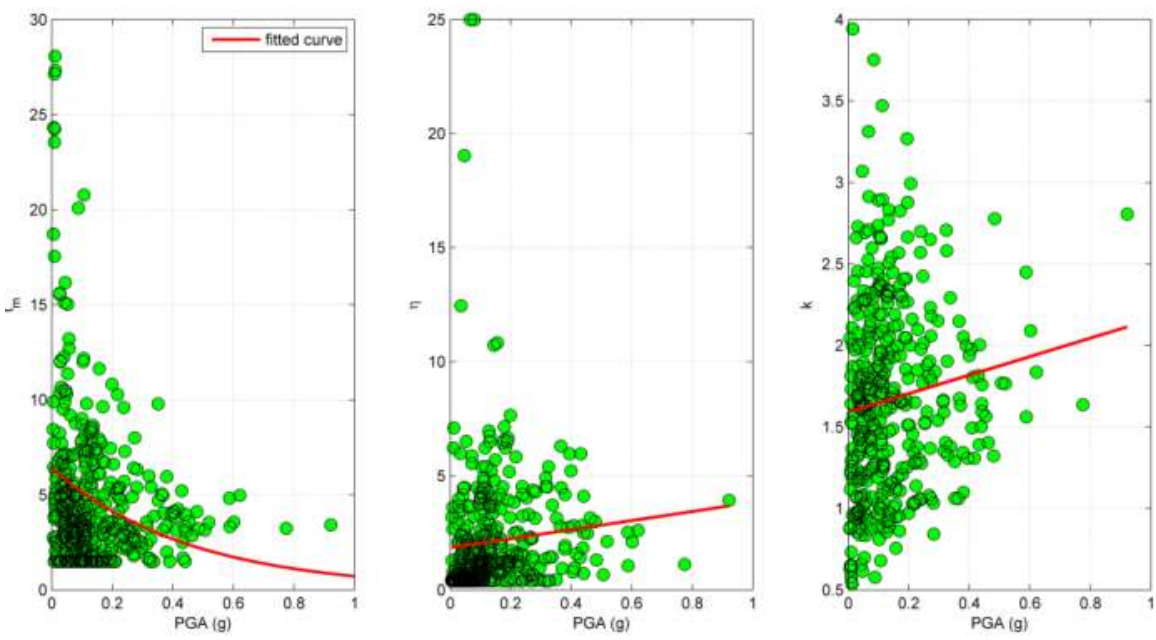

Figure 13. Values of $t_{m^{\prime}} \eta$ and $k$ defined as function of the PGA. The value are related to the set of the seismic events selected from the database and occurred in sites characterized by kind of soil D.

\section{Conclusions}

One of the main problems of earthquake engineering is the proper estimation of the characteristics of future earthquakes that will affect new and existing structures. This is a non-trivial problem because of the inner unpredictable nature of earthquakes. Due to this nature of earthquakes, stochastic models have been proposed to generate synthetic future seismic accelerograms to use in the structural design. Some of the stochastic models already proposed relate the stochastic ground motion process to seismological parameters that are not meaningful in structural engineering. The study here proposed overcomes this limit: it presents a model that describes the earthquake ground motion in term of parameters useful in the structural engineering. This model is a non-stationary stochastic one based on the stationary C-P model and characterized by the temporal modulation of the amplitude. The amplitude modulation is produced by a new envelope function that has the same shape of the $\mathrm{SH}$ function, but it is described by only two parameters. In order to obtain the values of these parameters of the envelope function a complex procedure is used. The procedure has two stages:

1. The estimation of parameters for each of selected accelerograms of the PEER Next Generation Attenuation database to generate a pre-envelope function of each accelogram.

2. The regression analysis of the values of these parameters to obtain their mean values for a class of soil. 
In order to estimate the values of the parameters of the envelope function for a class of soil other analytical results are obtained. Relations of the parameters of the MSH envelope function with other characteristics of the earthquake ground motions and the AI are imposed (eqs. (13), (19), (20) and (21)), so analytical formulae to estimate other characteristics of the seismic events in term of PGA and kind of soil are obtained from the regression analysis (eqs. (25), (26), (27), (28), (29)).

The envelope function here presented and the method to calculate its parameters produce a temporal modulation of the amplitude in the synthetic accelograms in term of the most significant quantities used in structural engineering: the PGA and the kind of soil.

Finally, the numerical values of the characteristics of the earthquake ground motion obtained from the regression analysis are collected in table to be used in future applications of the earthquake engineering.

\section{Author details}

Giuseppe Carlo Marano ${ }^{1}$, Mariantonietta Morga ${ }^{2}$ and Sara Sgobba ${ }^{1}$

1 Department of Civil and Architectural Science, Technical University of Bari, Bari, Italy

2 Mobility Department - Transportation Infrastructure Technologies, Austrian Institute of Technologies GmbH, Vienna, Austria

\section{References}

[1] Bolotin VV. Statistical theory of the aseismic design of structures. In: 2nd World Conference on Earthquake Engineering - Tokio, volume 2, pages 1365-1374. Science Council of Japan, 1960.

[2] Housner GW and Jennings PC. Generation of artificial earthquakes. ASCE, Journal of the Engineering Mechanics Division, 1964; 90: 113-150.

[3] Housner GW. Characteristics of strong-motion earthquakes. Bulletin of the Seismological Society of America, 1947; 37(1) 19-31.

[4] Kanai K. Semi-empirical formula for the seismic characteristics of the ground motion. Bulletin of the Earthquake Research Institute, 1957; 35: 309-325.

[5] Tajimi H. A statistical method of determining the maximum response of a building structure during an earthquake. In: 2nd World Conference on Earthquake Engineering - Tokio, volume 2, pages 781-798. Science Council of Japan, 1960. 
[6] Sgobba S, Marano GC, Stafford PJ, and Greco R. New Trends in Seismic Design of Structures, chapter: Seismologically consistent stochastic spectra. Saxe-Coburg Publisher, 2009.

[7] Clough RW and Penzien J. Dynamics of structures, Mc Graw Hill; 1975.

[8] Jangid RS. Response of SDoF system to non-stationary earthquake excitation. Earthquake Engineering \& Structural Dynamics, 2004; 33(15) 1417-1428.

[9] Campbell KW. Prediction of strong ground motion using the hybrid empirical method: example application to eastern-north America. Bulletin of the Seismological Society of America, 2002; 93(3) 1012-1033.

[10] Cua G. Creating the Virtual Seismologist: Developments in Ground Motion Characterization and Seismic Early Warning. PhD thesis, California Institute of Technology, 2005.

[11] Stafford PJ, Sgobba S, and Marano GC. An energy-based envelope function for the stochastic simulation of earthquake accelerograms. Soil Dynamics and Earthquake Engineering, 2009; 29(7) 1123-1133.

[12] Baker JW. Correlation of ground motion intensity parameters used for predicting structural and geotechnical response. In: ICASP10 - 10th International Conference on Applications of Statistics and Probability in Civil Engineering, Tokyo, Japan, 2007.

[13] Conte JP and Peng BF. Fully nonstationary analytical earthquake ground-motion model. ASCE, Journal of Engineering Mechanics, 1997; 12: 15-24.

[14] Saragoni GR and Hart GC. Simulation of artificial earthquake. Earthquake Engineering and Structural Dynamics, 1974; 2: 249-267.

[15] Marano GC, Morga M and Sgobba S. Modelling of stochastic process for earthquake representation as alternative way for structural seismic analysis: past, present and future. In: EQADS 2011 - International Conference on Earthquake Analysis and Design of Structures - Department of Civil Engineering, PSG College of Technology, Coimbatore, Tamilnadu, India, December 1-3 2011.

[16] Amin M and Ang AHS. Nonstationary stochastic model of earthquake motions. ASCE, Journal of the Engineering Mechanics Division, 1968; 94: 559-583. 